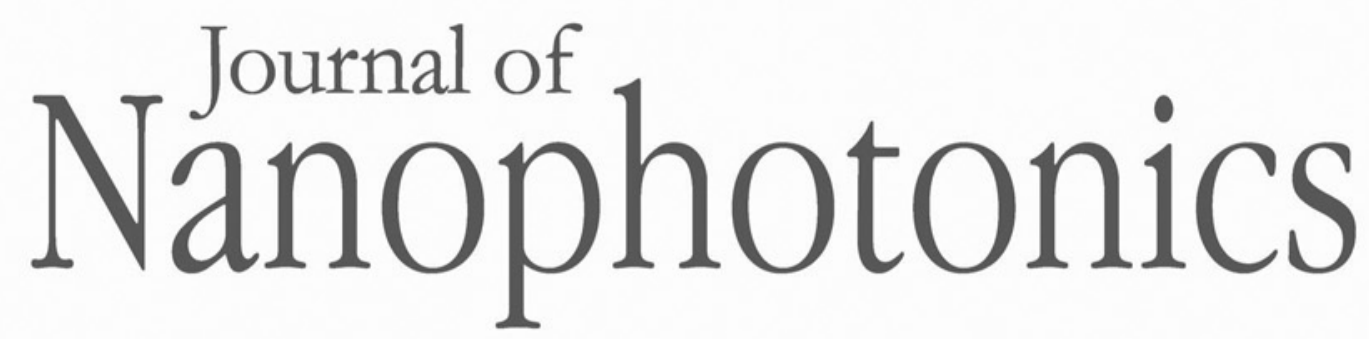

\title{
Synthesis and optoelectrical properties of f-graphene/cadmium selenide hybrid system
}

Saeed Salem Babkair

Ameer Azam

Kuldeep Singh

Sundeep Kumar Dhawan

Mohd Taukeer Khan

\section{SPIE.}




\title{
Synthesis and optoelectrical properties of f-graphene/cadmium selenide hybrid system
}

\author{
Saeed Salem Babkair, ${ }^{\text {a }}$ Ameer Azam, ${ }^{\text {b }}$ Kuldeep Singh, \\ Sundeep Kumar Dhawan, ${ }^{d}$ and Mohd Taukeer Khan ${ }^{\mathrm{e}, *}$ \\ ${ }^{a}$ King Abdulaziz University, Center of Nanotechnology, Department of Physics, \\ Faculty of Science, Jeddah 21589, Saudi Arabia \\ ${ }^{\mathrm{b}}$ Aligarh Muslim University, Department of Applied Physics, Aligarh-202002, India \\ ${ }^{\mathrm{c}}$ Central Electro Chemical Research Institute (CSIR), Karaikudi-630006, Tamil Nadu, India \\ ${ }^{d}$ National Physical Laboratory, (CSIR), Dr. K. S. Krishnan Road, New Delhi 110 012, India \\ eIslamic University, Department of Physics, Faculty of Science, Medina 42351, Saudi Arabia
}

\begin{abstract}
The present work demonstrates the synthesis of a hybrid accepter material containing amino-functionalized graphene oxide (GO) and an inorganic semiconducting material, cadmium selenide $(\mathrm{CdSe})$. First, amino-functionalized graphene was synthesized and then nanocrystals (NCs) of CdSe were in situ grown in the functionalized-(GO) matrix named f-GCdSe. Structural studies such as X-ray diffraction, and a scanning electron microscopic were employed to investigate the growth of $\mathrm{CdSe} \mathrm{NCs}$ in the graphene matrix. To understand the charge generation and transfer process at the donor/acceptor interface, the absorption, photoluminescence (PL), and transient absorption spectroscopic (TAS) studies have been carried out in poly(3-hexylthiophene) (P3HT)/f-GCdSe thin films. PL quenching in P3HT/f-GCdSe thin film suggests that charge transfer takes place at the donor/acceptor interface. TAS shows higher optical density and long lived free carriers for P3HT/f-GCdSe thin film. These results suggest that f-GCdSe is an excellent electron-acceptor material for organic photovoltaic devices. () 2015 Society of Photo-Optical Instrumentation Engineers (SPIE) [DOI: 10.1117/1.JNP.9.093048]
\end{abstract}

Keywords: graphene; cadmium selenide; nanostructure; acceptor; transient absorption spectroscopy; charge transfer.

Paper 15045 received Jun. 10, 2015; accepted for publication Sep. 11, 2015; published online Oct. 13, 2015.

\section{Introduction}

The past decade has witnessed a lot of advancement in the area of organic photovoltaic (OPV) technology by scientists and engineers. Recently, double digit power conversion efficiency has already been achieved by many groups. ${ }^{1-3}$ The main attractive aspect of OPV devices over traditional Si solar cells is their cost-effectiveness as they can be easily manufactured by roll-to-roll processes. In addition, OPV has a better performance in low light, can be transparent and colorful, and has greater flexibility and lower weight. However, the disadvantage of OPV is their low efficiency in comparison to traditional silicon solar cells. To make polymer-based solar cells an economically viable alternative, the efficiency of these devices needs to be increased. The limited efficiency of the OPV is due to the poor carrier mobility, ${ }^{4}$ short exciton diffusion length, ${ }^{5}$ charge trapping, ${ }^{6}$ and the mismatch of the absorption spectrum of the active layer and the solar emission. $^{7}$ To address these fundamental limitations of polymer solar cells, inorganic NCs are blended with organic materials which integrate the benefits of both classes of materials. Various NCs including $\mathrm{CdS},{ }^{8}$ cadmium selenide (CdSe), ${ }^{9,10} \mathrm{CdTe},{ }^{11} \mathrm{PbS},{ }^{12} \mathrm{ZnO},{ }^{13}$ and $\mathrm{TiO}_{2}{ }^{14}$ have been widely studied for hybrid solar cell fabrication. However, the efficiency of organic/inorganic hybrid solar cells reported so far is still less than 5\%, which is still far from the requirement for the next-generation solar cell for commercial applications. A major

*Address all correspondence to: Mohd Taukeer Khan, E-mail: khanmtk@gmail.com

1934-2608/2015/\$25.00 (C) 2015 SPIE

Journal of Nanophotonics

093048-1

Vol. 9, 2015 
challenge in developing high-performance organic/inorganic hybrid solar cells is the effective separation of photogenerated electron-hole pairs and the transfer of the electrons to the electrode.

Various strategies have been employed to enhance the separation of photogenerated electronhole pairs in hybrid devices. One way to improve charge separation is to develop an organic/inorganic nanocomposite. It has been observed that $\mathrm{C}_{60}$ played an effective role in the capture and transfer of photogenerated electrons in a $\mathrm{CdSe}-\mathrm{C}_{60}$ composite employed in quantum dot (QD) sensitized solar cells. ${ }^{15}$ Similar results have also been reported with CdS-CNT nanostructure as the light-harvesting assembly. ${ }^{16}$ Compared with $\mathrm{C}_{60}$ and CNT, graphene, a monolayer of $\mathrm{sp}^{2}$-hybridized carbon atoms arranged in an archetypal two-dimensional (2-D) lattice, is more efficient for the application in OPV due to its supreme structural and electrical properties. ${ }^{17}$ The high surface area, superior room temperature electron mobility of the order of $2.5 \times 10^{5} \mathrm{~cm}^{2} \mathrm{~V}^{-1} \mathrm{~s}^{-1},{ }^{18}$ and tunable bandgap make it an excellent electron acceptor in OPV devices. ${ }^{19}$ The high carrier mobility of graphene can facilitate electron transport to the photoanode, thereby decreasing the probability for carrier recombination and extending its lifetime. ${ }^{20,21}$ The high surface area provides large donor/acceptor interface for charge separation. Moreover, it is well known that high loading of nanocrystals (NCs) is necessary for better charge transport in organic/inorganic hybrid solar cells. Consequently, excessive volume fraction increases the risk of aggregation. In functionalized-graphene (f-G), the functional groups can act as anchor sites for inorganic NCs and prevent their aggregation. ${ }^{22}$ Furthermore, modification of the graphene surface with QDs causes fluorescence quenching due to the electrons flowing from the conduction band of the QD to the graphene sheets. ${ }^{23}$ Therefore, the hybrid nanostructures based on graphene can be considered as the potential acceptor material for organic solar cells.

Hence, it is of great interest and importance to bind CdSe NCs onto graphene because the combination and interaction between NCs and graphene will lead to multifunctional or even completely new properties in such a nanocomposite. ${ }^{24,25}$ Herein, we report the in situ synthesis of CdSe NCs in a functionalized graphene matrix, wherein the strategy of in situ growing CdTe NCs onto a P3HT matrix has been adopted. ${ }^{11}$ The f-GCdSe nanocomposite shows a very fast and dramatically enhanced photoresponse, which makes it an excellent electron-acceptor material for OPV devices.

\section{Synthesis of Functionalized-Graphene/Cadmium Selenide Nanocomposites}

Prior to in-situ synthesis of CdSe NCs in functionalized graphene, an aqueous suspension of graphene oxide (GO) was synthesized from natural graphite powder using modified Hummer's method. ${ }^{26}$ In a typical process, 3.5 g graphite was charged into a $500 \mathrm{ml}$ beaker and heated for $10 \mathrm{~s}$ in a microwave oven to expand the graphite powder. The powder was mixed with $500 \mathrm{ml}$ concentrated $\mathrm{H}_{2} \mathrm{SO}_{4}$ at $0^{\circ} \mathrm{C}$ with continuous stirring. Then $21 \mathrm{~g}$ of $\mathrm{KMnO}_{4}$ was slowly added and maintained below $20^{\circ} \mathrm{C}$. The temperature was then increased to $35^{\circ} \mathrm{C}$, and then $70^{\circ} \mathrm{C}$ for $2 \mathrm{~h}$ with continuous stirring. The flask was then chilled again in the ice bath, and distilled water (ice) was slowly added, maintaining the temperature below $70^{\circ} \mathrm{C}$. The mixture was stirred for $1 \mathrm{~h}$ and subsequently diluted with $5 \mathrm{~L}$ of deionized water. A $50 \mathrm{ml}$ of $\mathrm{H}_{2} \mathrm{O}_{2}(30 \mathrm{wt}$. $\%$ ) was added, and vigorous bubbles appeared as the color of the suspension changed from dark brown to yellow. The suspension was centrifuged and washed several times with $10 \% \mathrm{HCl}$ solution until the $\mathrm{pH}$ of the GO dispersion reached 6. The as-synthesized GO dispersion was a paste with a concentration of $1.0 \mathrm{wt}$ \% GO. The calculated amount of GO was then treated with $\mathrm{SOCl}_{2}$ in dimethylformamide followed by octadecylamine (ODA) at $150^{\circ} \mathrm{C}$ for $24 \mathrm{~h}$ to yield functionalized graphene. The in situ synthesized CdSe NCs in graphene were named as f-GCdSe and are schematically illustrated in Fig. 1. In a typical synthesis of f-GCdSe, $100 \mathrm{mg}$ of functionalized graphene has been allowed to react with $0.1 \mathrm{mmol}$ of cadmium acetate dihydrate at $150^{\circ} \mathrm{C}$ in the medium of ODA and the reaction temperature was increased to $300^{\circ} \mathrm{C}$ for $2 \mathrm{~h}$. The selenium precursor was prepared by treating $0.2 \mathrm{mmol}$ of selenium powder (Acros Organics) in trioctylphosphine (Sigma Aldrich), at $300^{\circ} \mathrm{C}$ for $30 \mathrm{~min}$ under nitrogen flow. The Se precursor was then injected into the functionalized graphene-Cd solution and the mixture was allowed to react for 


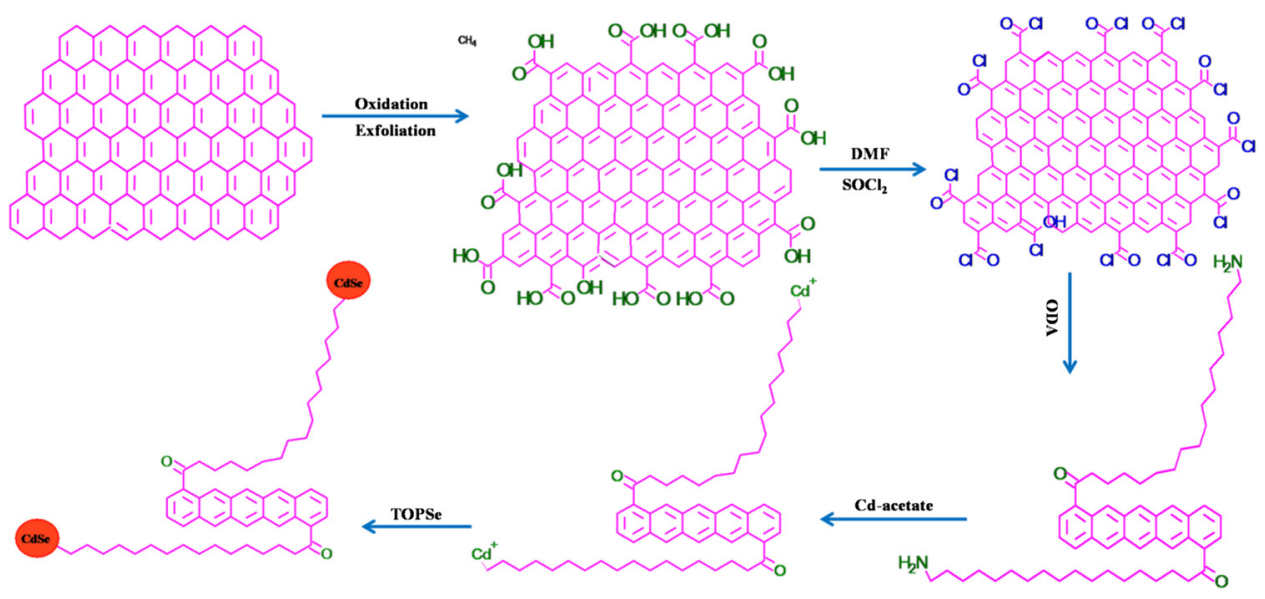

Fig. 1 Schematic illustration of the in situ growth of the cadmium selenide (CdSe) nanocrystals (NCs) in the f-graphene oxideP3HT matrix.

$30 \mathrm{~min}$ at $300^{\circ} \mathrm{C}$ under nitrogen flow. The growth of CdSe NCs is completed when the color of the solution turns to dark orange. After the completion of the reaction, the unreacted cadmium acetate and precursor of Se were removed by treating the nanocomposites with hexane. The reaction mixture was separated by centrifugation and dried in vacuum at $40^{\circ} \mathrm{C}$. Similarly, another composition of functionalized graphene containing a different molar ratio of Cd-acetate was synthesized and was designated as f-GCdSe2 of $0.2 \mathrm{mmol}$.

\section{Results and Discussion}

\subsection{Structural Study}

The XRD patterns of nanocomposite powder were recorded using Rigaku Ultima X-ray diffractometer $\left(40 \mathrm{KV}, 30 \mathrm{~mA}, \lambda=1.54059 \mathrm{~A}, \mathrm{Cu}-\mathrm{K}_{\alpha 1}\right)$ and are shown in Fig. 2. The XRD pattern of the $\mathrm{f}-\mathrm{G}$ sample shows a broad peak at $20 \mathrm{deg}$. The broad diffraction peak of $\mathrm{f}-\mathrm{G}$ can be attributed to two factors: first, the small sheet size ( $1 \mu \mathrm{m}$ and below) and, second, a relatively short domain order or turbostratic arrangement of f-GO stacked sheets, each of which broadens the XRD peak. $^{27}$ In comparison, XRD data of f-GCdSe show an additional peak at $2 \theta-25.2 \mathrm{deg}$, $42.4 \mathrm{deg}$, and $49.5 \mathrm{deg}$ which is ascribed to the presence of CdSe NCs in the $\mathrm{f}-\mathrm{G}$ matrix.

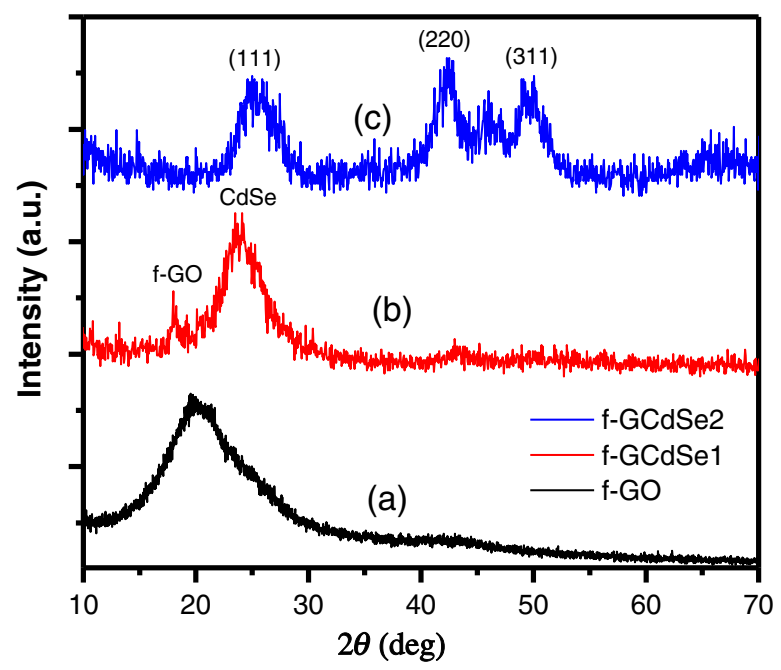

Fig. 2 X-ray diffraction (XRD) spectra of: (a) f-graphene oxide (f-GO), (b) f-GCdSe1, and (c) f-GCDSe2 nanocomposites. 
These peaks at 20-25.2 deg, $42.4 \mathrm{deg}$, and $49.5 \mathrm{deg}$ correspond to the (111), (220), and (311) planes, respectively, of the zinc blend phase of $\mathrm{CdSe} .{ }^{28}$ The XRD peaks are broadened due to the small size of CdSe NCs. In the XRD spectra of f-GCdSe, the low intensity of the CdSe peaks at $2 \theta-42.4 \mathrm{deg}$ and $49.5 \mathrm{deg}$ may be attributed to the low concentration of CdSe in f-GO. These results are in good agreement with scanning electron microscopy (SEM) micrographs shown in Fig. 3(a). On the other hand, the peak at 20-19 deg in f-GCdSe2 almost disappears due to the higher concentration of $\mathrm{CdSe}$ in $\mathrm{f}-\mathrm{GCdSe}$, which is also evident in Fig. 3(b). The nanoscale morphology is a crucial parameter to understand the effectiveness of the interface for exciton splitting into free charge carriers and the formation of a percolation network for efficient transport of charge carriers to the electrodes. The surface morphologies of the f-GCdSe1 and f-GCdSe2 nanocomposite films have been examined by SEM and are presented in Fig. 3. These SEM micrographs were observed by a field emission SEM (Jeol FESEM-7600F). It is quite clear that $\mathrm{CdSe} \mathrm{NCs}$ are evenly distributed within the hybrid system with a low CdSe NCs content [Fig. 3(a)], while NCs are massively phase separated or aggregated from the f-GO matrix at the higher NCs content [Fig. 3(b)]. An even more drastic contrast between organic and inorganic phases can be observed in f-GCdSe2.

\subsection{Raman Analysis}

Raman spectroscopy was performed on a Thermoscientific Raman microscope (DXR) under 532-nm excitation at low power levels to avoid laser heating. The Raman spectra of f-GO and f-GCdSe nanocomposites are shown in Fig. 4. f-GO, fGCdSe1, and fGCdSe2 exhibit characteristic D-bands at 1342, 1346, and $1343 \mathrm{~cm}^{-1}$ and G-bands at 1583, 1578, and $1576 \mathrm{~cm}^{-1}$, respectively. The D-band may be assigned to local defects and disorders, whereas the G-band originates from symmetric stretching of the $\mathrm{sp}^{2} \mathrm{C}-\mathrm{C}$ bond. ${ }^{29}$ It can be clearly noticed that the G-band of the fGCdSe nanocomposite shows a red shift in comparison to the f-GO. This shift indicates the interaction between graphene and CdSe nanoparticles, which is a prerequisite for charge transfer between CdSe and graphene. ${ }^{30}$ The shape and position of the 2D-band dramatically change on incorporating CdSe into f-GO, as shown in Fig. 4. The 2D-band in fGCdSe can be resolved into two or more components, whereas the monolayer f-GO has a single component. According to this graph, it can also be seen that the symmetric and sharp 2D-band at $2677 \mathrm{~cm}^{-1}$ is the best indicator for less than five layers of graphene. ${ }^{31}$ The center of the 2D-band of fGCdSe nanocomposites shows a blue shift and can be resolved into two or more components, which further confirms the incorporation of CdSe nancrystals in a graphene matrix.

\subsection{Spectroscopic Properties}

The sample for spectroscopy measurement was prepared by spin-coating of polymer solution onto a glass substrate at $2000 \mathrm{rpm}$ for $60 \mathrm{~s}$ and was then annealed at $393 \mathrm{~K}$ for $30 \mathrm{~min}$. Absorption spectra were taken on a Shimadzu UV-1601 spectrophotometer. The normalized

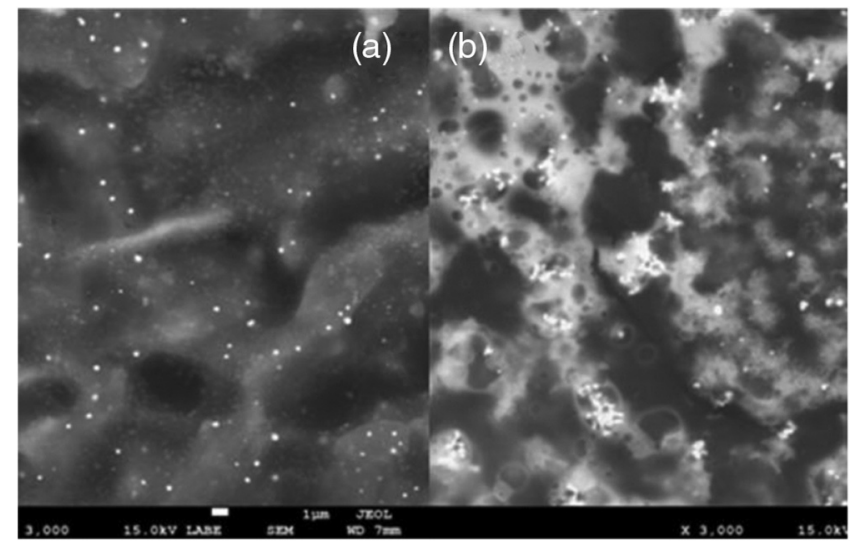

Fig. 3 Scanning electron microscope (SEM) micrograph of (a) f-GCdSe1 and (b) f-GdSe2. 


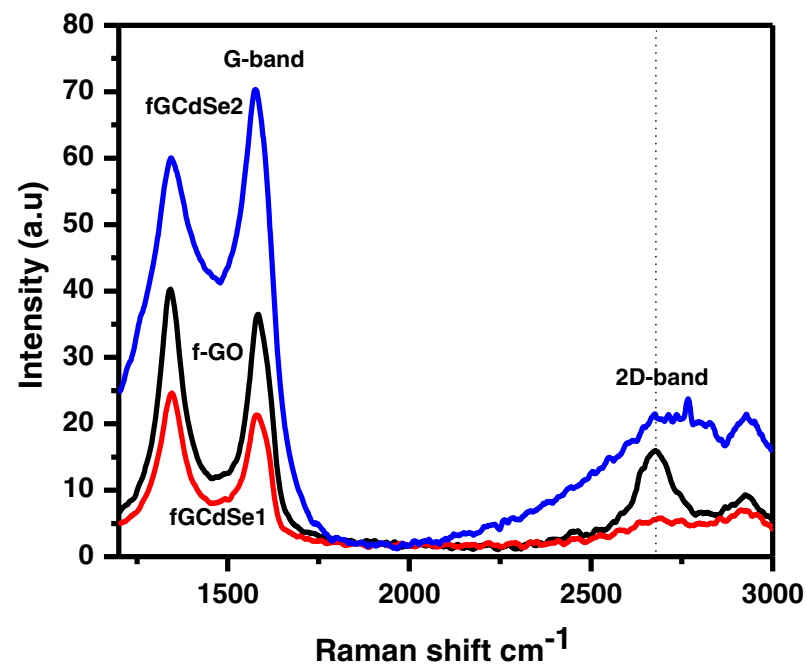

Fig. 4 Raman spectrum of the f-GO, fGCdSe on $\mathrm{Si} / \mathrm{SiO} 2$ substrate.

UV-Vis absorption spectra of the nanocomposites' thin films with different concentrations of $\mathrm{f}-G C \mathrm{dSe} 2$ in P3HT are shown in Fig. 5. The maximum absorption of pristine P3HT films was observed at $522 \mathrm{~nm}$, which corresponds to the $\pi-\pi^{*}$ transition of the conjugated chain in the P3HT. ${ }^{32}$ It can be seen that the P3HT/f-GCdSe thin films have almost the same absorption range and peaks as that of P3HT film in the wavelength range from 350 to $650 \mathrm{~nm}$, with the exception of a broad band that occurs in the blue region as compared to pristine P3HT. This is caused by the absorption of CdSe NCs in this region as evidenced from absorption spectra of f-GCdSe shown in the inset of Fig. 5. The absorption spectrum of the P3HT/f-GCdSe shows no significant change as compared to pristine P3HT, which implies that in the P3HT/f-GCdSe thin films, no significant ground-state interactions take place between the two materials, and hence no charge transfer occurs in the ground state.

For bulk heterojunction photovoltaic cells, photo-induced charge transfer from the donor polymer to the acceptor is usually needed for the photocurrent generation. Photoluminescence (PL) in conjugated P3HT is well known to arise from radiative recombination of polaron-exciton pairs into Franck-Condon (FC) states, ${ }^{33}$ and PL quenching of an appropriate donor polymer by

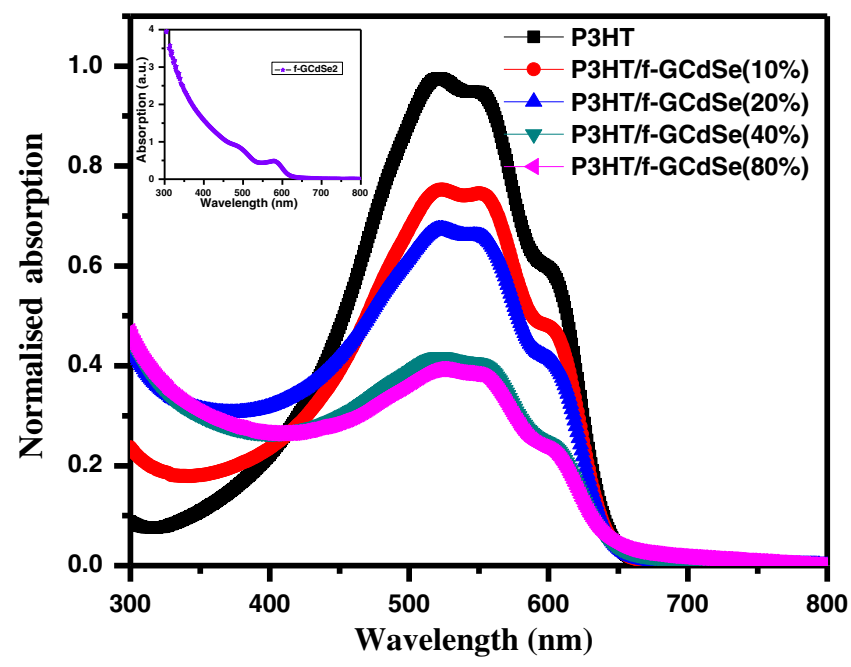

Fig. 5 Normalized absorption spectra of P3HT and P3HT/f-G-CdSe nanocomposite films with different concentrations of the f-G-CdSe in P3HT. Inset shows absorption of f-GCdSe solution dissolved in tetrahydrofuran. Percentage of $\mathrm{f}-\mathrm{GCdSe}$ in $\mathrm{P} 3 \mathrm{HT}$ is mentioned in bracket. 
a suitable acceptor gives an indication of an effective photo-induced charge transfer from the donor to the acceptor. ${ }^{34}$ In Fig. 6, the PL spectra of pristine P3HT film have been compared with that of different nanocomposites' films containing 20\%, 40\%, 60\%, and $80 \%$ of f-GCdSe in P3HT. These spectra were taken on an A Shimadzu RF-5301PC with an excitation wavelength of $525 \mathrm{~nm}$. It can be observed from Fig. 6 that the P3HT thin film shows strong PL between 600 and $800 \mathrm{~nm}$. The PL intensity of the nanocomposite films is significantly reduced at $40 \%$ concentration of f-GCdSe in polymer. With the increase of f-GCdSe concentration in polymer, the PL intensity decreases further. The reduced PL intensity of the composites relative to the pristine P3HT indicates that the charge transfer, and thus the exciton dissociation at the interface of f-GCdSe and P3HT. ${ }^{11}$ These results show that the excited fluorophore in the P3HT backbone is quenched by the electronic interactions at the P3HT and f-GCdSe interfaces. By referencing previous work with $\mathrm{PCBM}^{34}$ and carbon nanotubes,${ }^{35}$ this efficient quenching of the PL emission shows that $\mathrm{f}-\mathrm{GCdSe}$ is expected to be an effective electron-acceptor material for OPV applications.

Figure 7(a) shows the transient absorption spectra (TAS) of P3HT, P3HT/f-G, and P3HT/ f-GCdSe thin films. A GL-301 PTI dye laser module was used in conjunction with the PTI GL3300 nitrogen laser to provide a variable wavelength source of excitation (400 to $750 \mathrm{~nm}$ ). It is clear from the figure that P3HT/f-GCdSe films have a very high optical density $(\Delta \mathrm{OD})$ as compared to pristine $\mathrm{P} 3 \mathrm{HT}$ as well as $\mathrm{P} 3 \mathrm{HT} / \mathrm{f}-\mathrm{G}$. In the P3HT film, only the polarons generated by interchain charge separation contribute to the mid-IR signal. ${ }^{36}$ In the composite, both the products of interchain charge separation (polarons in polymer) and interfacial electron transfer (positive polarons in polymer and injected electrons in $\mathrm{CdSe}$ ) can contribute to the observed IR signal. The enhanced optical density of the composite film indicates photo-induced charge separation resulting in an increase of the yield for free carrier generation, and a higher sum of the mobilities of free carriers because of the contribution of the electron mobility in the f-GCdSe. Charge separation will also result in longer photocarrier lifetimes due to the electron and the hole residing in two different phases in the composite material. P3HT decay is short lived and appears crudely in the first order with a half life of approximately $1.2 \mu \mathrm{s}$, while the composite signal decay continues over 5 orders of magnitude in time up to $10 \mathrm{~ms}$. Furthermore, the average emission lifetimes of P3HT, P3HT/f-GO, and P3HT/f-GCdSe are $0.5,0.75$, and $0.69 \mu$ s, respectively. The increase in the lifetime for P3HT/f-GO compared with P3HT indicates that there is photogenerated electron transport from P3HT to f-GO. On the other hand, the lifetime for P3HT/ fGCdSe decreases from 0.75 to $0.69 \mu \mathrm{s}$, which may be attributed to electron transfer from P3HT to CdSe and then to graphene as shown in Fig. 7(b).

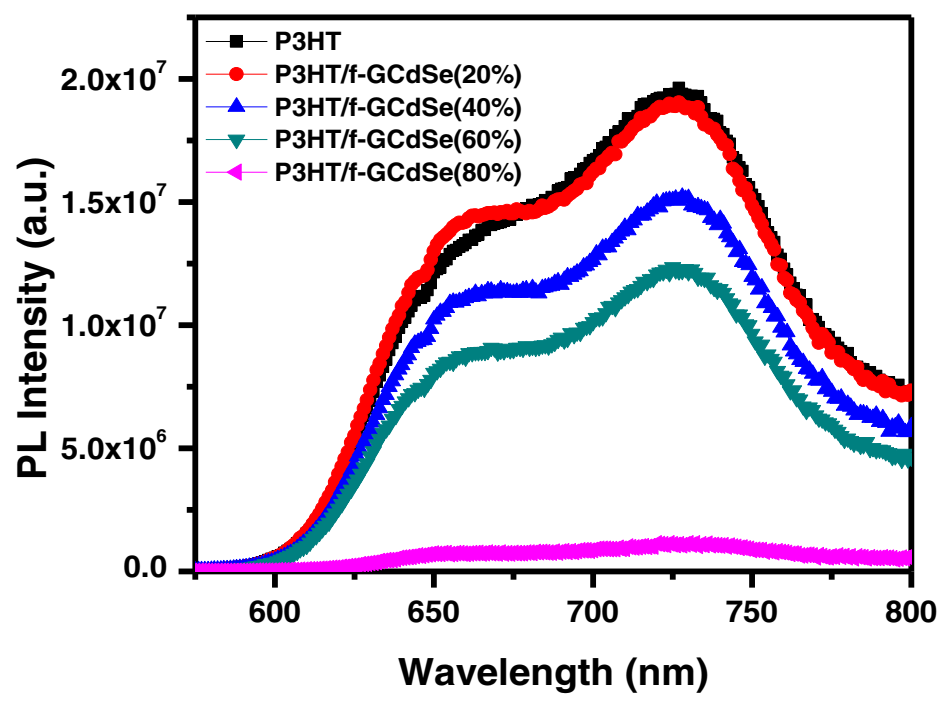

Fig. 6 Photoluminescence (PL) spectra of P3HT, P3HT/f-G-CdSe nanocomposite thin films after excitation by radiation of $525-\mathrm{nm}$ wavelengths. 

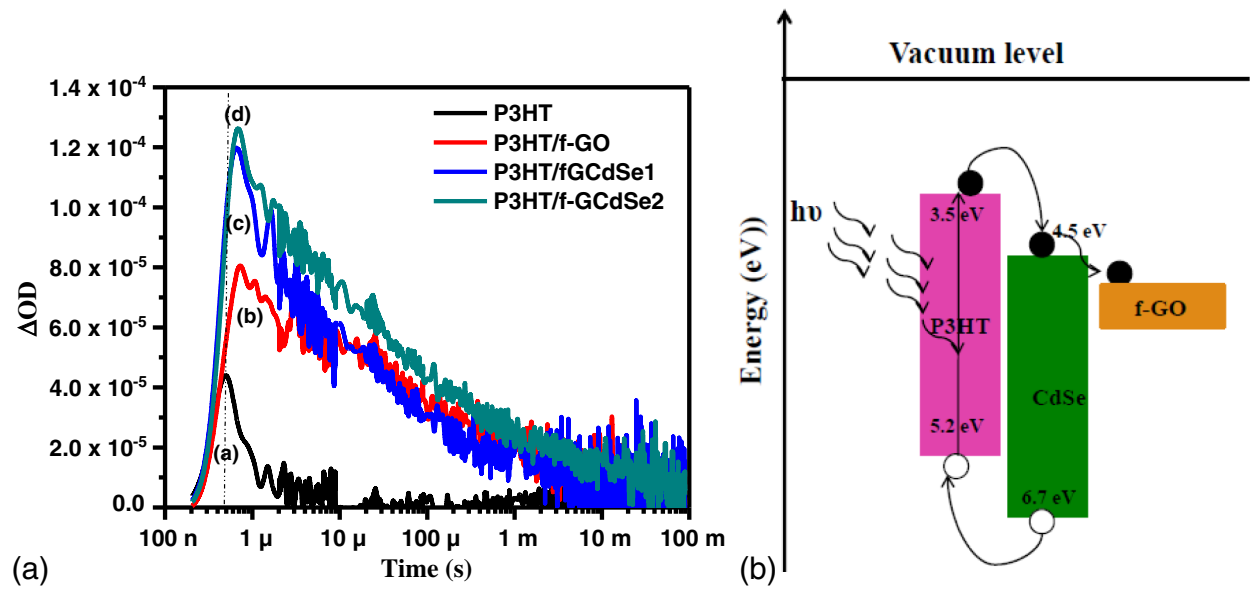

Fig. 7 (a) Transient absorption spectroscopy (TAS) of (a) P3HT, (b) P3HT/f-GO, (c) P3HT/ f-GCdSe1 and (d) P3HT/f-GCdSe2 thin films. (b) Schematic illustration of the charge transfer at the P3HT-CdSe-f-GO interface.

\section{Conclusions}

In conclusion, the successful in situ incorporation of CdSe NCs in a reduced graphene matrix has been demonstrated. Structural and morphological studies reveal that CdSe NCs are uniformly distributed in the carbon matrix. PL quenching in P3HT/f-GCdSe suggests that charge transfer takes place between P3HT and f-GCdSe NCs. Charge transfer between donor and acceptor is further confirmed by time resolved absorption spectroscopy, which shows higher optical density and long lived free charge carriers. These results suggest that f-GCdSe is an excellent electronacceptor material for OPV devices.

\section{References}

1. J. You et al., "A polymer tandem solar cell with $10.6 \%$ power conversion efficiency," Nat. Commun. 4(1446), 1-10 (2013).

2. M. Peach, "Heliatek achieves $12 \%$ organic solar cell efficiency," 23 January 2013, http:// optics.org/news/4/1/36.

3. Y. Liu et al., "Solution-processed small-molecule solar cells: breaking the $10 \%$ power conversion efficiency," Sci. Rep. 3(3356), 1-8 (2013).

4. S. Shao et al., "High-efficiency hybrid polymer solar cells with inorganic P- and N-type semiconductor nanocrystals to collect photogenerated charges," J. Phys. Chem. C 114(19), 9161-9166 (2010).

5. S. R. Scully and M. D. McGehee, "Effects of optical interference and energy transfer on exciton diffusion length measurements in organic semiconductors," J. Appl. Phys. 100(3), 034907 (2006).

6. F. Yang, M. Shtein, and S. R. Forrest, "Controlled growth of a molecular bulk heterojunction photovoltaic cell," Nat. Mater. 4(1), 37-41 (2005).

7. M. M. Wienk et al., "Low-band gap poly(di-2-thienylthienopyrazine): fullerene solar cells," Appl. Phys. Lett. 88(15), 153511-1535113 (2006).

8. M. T. Khan et al., "Effect of cadmium sulphide quantum dot processing and post thermal annealing on P3HT/PCBM photovoltaic device," Thin Solid Films 519(3), 1007-1011 (2010).

9. S. Dayal et al., "Photovoltaic devices with a low band gap polymer and CdSe nanostructures exceeding 3\% efficiency," Nano Lett. 10(1), 239-242 (2010).

10. Y. Zhou et al., "Improved efficiency of hybrid solar cells based on non-ligand-exchanged CdSe quantum dots and poly (3-hexylthiophene)," Appl. Phys. Lett. 96(1), 01330410133043 (2010). 
11. M. T. Khan et al., "In-situ growth of cadmium telluride nanocrystals in poly(3-hexylthiophene) matrix for photovoltaic application," J. Appl. Phys. 110(4), 0445091-0445097 (2011).

12. N. Zhao et al., "Colloidal PBS quantum dot solar cells with high fill factor," ACS Nano 4(7), 3743-3752 (2010).

13. S. D. Oosterhout et al., "The effect of three-dimensional morphology on the efficiency of hybrid polymer solar cells," Nat. Mater. 8(10), 818-824 (2009).

14. S. Ren et al., "Heterojunction photovoltaics using GaAs nanowires and conjugated polymers," Nano Lett. 11(2), 408-413 (2011).

15. P. Brown and P. V. Kamat, "Quantum dot solar cells. Electrophoretic deposition of CdSeC60 composite films and capture of photogenerated electrons with n C60 cluster shell," J. Am. Chem. Soc. 130(28), 8890-8891 (2008).

16. I. Robel, B. A. Bunker, and P. V. Kamat, "Single-walled carbon nanotube-CdS nanocomposites as light-harvesting assemblies: photoinduced charge-transfer interactions," Adv. Mater. 17(20), 2458-2463 (2005).

17. K. S. Novoselov et al., "A roadmap for graphene," Nature 490(7419), 192-200 (2012).

18. A. S. Mayorov et al., "Micrometer-scale ballistic transport in encapsulated graphene at room temperature," Nano Lett. 11(6), 2396-2399 (2011).

19. Z. F. Liu et al., "Organic photovoltaic devices based on a novel acceptor material: graphene," Adv. Mater. 20(20), 3924-3930 (2008).

20. S. R. Sun, L. Gao, and Y. Q. Liu, "Enhanced dye-sensitized solar cell using graphene-TiO2 photoanode prepared by heterogeneous coagulation," Appl. Phys. Lett. 96(8), 08311310831133 (2010)

21. P. V. Kamat, "Graphene-based nanoarchitectures. Anchoring semiconductor and metal nanoparticles on a two-dimensional carbon support," J. Phys. Chem. Lett. 1(2), 520-527 (2010).

22. S. W. Tong et al., "High-performance hybrid solar cell made from CdSe/CdTe nanocrystals supported on reduced graphene oxide and PCDTBT," Adv. Funct. Mater. 24(13), 19041910 (2014).

23. J. Chen et al., "Flexible photovoltaic cells based on a graphene-CdSe quantum dot nanocomposite," Nanoscale 4(2), 441-443 (2012).

24. A. N. Cao et al., "A facile one-step method to produce graphene-CdS quantum dot nanocomposites as promising optoelectronic materials," Adv. Mater. 22(1), 103-106 (2010).

25. X. Geng et al., "Aqueous-processable noncovalent chemically converted graphene-quantum dot composites for flexible and transparent optoelectronic films," Adv. Mater. 22(5), 638-642 (2010).

26. W. S. Hummers and R. E. Offeman, "Preparation of graphitic oxide," J. Am. Chem. Soc. 80(6), 1339-1339 (1958).

27. S. Dubin et al., "A one-step, solvothermal reduction method for producing reduced graphene oxide dispersions in organic solvents," ACS Nano 4(7), 3845-3852 (2010).

28. N. A. Hamizi, C. S. Ying, and M. R. Johan, "Synthesis with different Se concentrations and optical studies of CdSe quantum dots via inverse Micelle technique," Int. J. Electrochem. Sci. 7(1), 4727-4734 (2012).

29. D. Graf et al., "Spatially resolved Raman spectroscopy of single-and few-layer graphene," Nano Lett. 7(2), 238-242 (2007).

30. H. Yu et al., "Graphene/polyaniline nanorod arrays: synthesis and excellent electromagnetic absorption properties," J. Mater. Chem. 22(40), 21679-21685 (2012).

31. I. Calizo et al., "Spectroscopic Raman nanometrology of graphene and graphene multilayers on arbitrary substrates," J. Phys. Conf. Ser. 109(1), 012008 (2008).

32. R. D. McCullough, "The chemistry of conducting polythiophenes (pages 93-116)," Adv. Mater. 10(2), 93-116 (1998).

33. B. Xu and S. Holdcroft, "Molecular control of luminescence from poly (3-hexylthiophenes)," Macromolecules 26(17), 4457-4460 (1993).

34. M. Al-Ibrahim et al., "Flexible large area polymer solar cells based on poly (3-hexylthiophene)/fullerene," Sol. Energy Mater. Sol. Cells 85(1), 13-20 (2005). 
35. S. Berson et al., "Elaboration of P3HT/CNT/PCBM composites for organic photovoltaic cells," Adv. Funct. Mater. 17(16), 3363-3370 (2007).

36. X. Ai et al., "Ultrafast photoinduced charge separation dynamics in polythiophene/SnO2 nanocomposites," J. Phys. Chem. B 110(50), 25496-25503 (2006).

Saeed Salem Babkair is an associate professor in the Department of Physics and the former deputy director of the Center of Nanotechnology at King Abdulaziz University, Jeddah, Saudi Arabia. He received his BSc degree from King Abdulaziz University and his MSc degree from Wright State University, Dayton, Ohio, USA, and his PhD from University of Salford, England (1988). His current research interests include energy conversion such as dye-sensitized solar cells, synthesis and properties of graphene-based materials, and nanostructured materials.

Ameer Azam is working as professor in the Department of Applied Physics, Faculty of Engineering \& Technology, Aligarh Muslim University, Aligarh, India. He has also worked as an associate professor in the Centre of Nanotechnology, King Abdulaziz University, Jeddah, Saudi Arabia for about four years (2010-2014). He has published 165 papers and supervised many for PhD, MPhil, and MTech theses. His research is focused on the multidisciplinary area of nanotechnology.

Kuldeep Singh is currently working as a scientist at CSIR-Central Electrochemical Research Institute, Karaikudi, and Tamilnadu, India. He has carried out his postdoctoral research under the supervision of Prof. Jin Suk Chung at the School of Chemical Engineering and Bioengineering, University of Ulsan, Republic of Korea Institute. His current research interests focus on design and synthesis of nano engineered low dimensional structure materials including conducting polymers, metal oxides, and graphene-based composites for energy storage devices and EMI shielding.

Sundeep Kumar Dhawan works as chief scientist in the National Physical Laboratory, New Delhi. His research activities are focused on design and modification of conducting polymers, nanoferromagnetic composites, graphene for EMI shielding, organic electronics, recent conducting polymer/matrix and designing of Intelligent coatings. He has published 160 papers, 6 US patents, and 8 Indian patents. He has received the outstanding scientist award in 2009 and DST-Lockheed Innovation Award in 2014 for his contribution in self-healing coatings.

Mohd Taukeer Khan is an assistant professor at the Islamic University of Medinah. His research focus is on charge transport mechanisms in organic semiconductors-based devices. He obtained his $\mathrm{PhD}$ in organic solar cells from the National Physical Laboratory New Delhi, India. He has worked as a postdoctoral researcher at the University of Leeds in the field of organic spintronics. His main research interest is to understand the spin dependent charge transport in organic semiconductors. 